\title{
La motivation autodéterminée des étudiant-es universitaires inscrits à un SPOC : quel type de régulation? Cas de la Faculté des Sciences de Rabat
}

\author{
Souhad Shlaka, Université Ibn Tofail
}

DOI : 10.51186/journals/ed.2020.10-2.e344

\section{Résumé}

La présente étude s'inscrit dans le cadre d'un projet de recherche portant sur l'impact du dispositif de formation de type Small Private Online Course (SPOC) sur la motivation des étudiant-es universitaires marocain-es. Nous exposons l'expérience d'un SPOC portant sur la terminologie scientifique, destiné aux étudiant-es ( $\mathrm{N}=792)$ inscrit-es en première année (S1) à la Faculté des Sciences de Rabat (FSR) et programmé dans le cadre du module «Langue et Terminologie ». Le cours a été conçu par une équipe d'enseignant-es chercheurs à la FSR et dispensé à travers la plateforme OpenEdx de l'Université Mohamed V (mooc.um5.ac.ma). Nous cherchons dans le cadre de cette étude à déterminer l'impact d'un dispositif de formation en ligne de type SPOC sur la motivation autodéterminée des étudiant-es. Au niveau du cadre théorique, la motivation a été abordée du point de vue de la théorie de l'autodétermination (TAD) de Deci \& Ryan (2000). Le choix d'une méthodologie de recherche mixte s'est traduit par l'administration d'un questionnaire en fin de SPOC, basé sur l'Échelle de Motivation en Formation d'Adulte (EMFA), complétée par l'exploitation des traces des étudiant-es sur la plateforme du cours. Un peu moins d'un tiers des étudiant-es ( $N=244)$ a répondu au questionnaire. L'analyse des données statistiques révèle que la motivation des étudiant-es est en grande partie contrôlée par des demandes externes qui s'apparentent à la décision de l'enseignant-e et à la récompense de la note obtenue en fin de cours. Ces résultats contredisent les traces des étudiant-es sur la plateforme qui attestent d'un grand engagement et de nombreuses initiatives d'activité.

Mots-clés : SPOC, apprentissage en ligne, étudiant-es universitaires, motivation autodéterminée, EMFA

\section{Abstract}

This study is part of a research project on the impact of a SPOC on the motivation of Moroccan university students. It presents the case study of a SPOC on scientific terminology for students $(\mathrm{N}=792)$ enrolled in the first year (S1) at the Faculty of Sciences of Rabat (FSR). The course, which is part of the module "Language and Terminology", was designed by a team of research teachers at the FSR and is delivered through the Mohamed V University's Openedx platform 
(mooc.um5.ac.ma). The study aims to determine the impact of a SPOC on students' selfdetermined motivation. At the theoretical level, motivation was approached from the point of view of Deci and Ryan's Theory of Self-determination (SDT). The study employed a mixedmethods research design that combined the administration of a questionnaire based on the Adult Education Motivation Scale (EMFA) at the end of the SPOC with the analysis of student traces on the course platform. The statistical analysis revealed that the motivation of students taking a SPOC as part of the university curriculum is largely controlled by external demands relating to the teacher's decision and the reward of the grade obtained at the end of the course.

Keywords: SPOC, online learning, university students, self-determined motivation, EMFA

\section{INTRODUCTION}

À l'instar des universités internationales, l'intégration du numérique est devenue une nécessité et une vision qui commence à prendre de l'ampleur dans le paysage de l'enseignement supérieur marocain. Dans cette optique, les orientations actuelles du Ministère de l'Enseignement Supérieur Marocain visent à promouvoir le numérique dans ledit secteur comme un levier créateur de la valeur de l'enseignement supérieur en vue de conduire le pays vers la société du savoir. Par ailleurs, des efforts ont été déployés notamment par l'ouverture à des coopérations et des partenariats internationaux afin d'initier davantage les enseignant-ees aux pratiques numériques pour que ces derniers se mettent à la page, en numérisant leurs pratiques au sein des universités. Ainsi, plusieurs projets ont été financés et mis en œuvre, visant à renforcer les équipements, à développer des contenus numériques et à améliorer la gestion administrative et pédagogique au sein des établissements d'enseignement supérieur. De leur côté, les universités ont concrétisé ces projets numériques notamment par la création de centres propres à chaque université et dédiés à la conception et l'élaboration de cours en ligne au profit des étudiant-es marocain-es et ce, afin de consolider le présentiel, dans la pénurie de ressources humaines (enseignant-es) et structurelles (locaux) pour rendre le savoir plus accessible à tous.

Dans cette dynamique, les MOOCs (Massive Open Online Courses) semblent séduire davantage les décideurs marocains. Ce choix est justifié par la montée remarquable des MOOCs dans les universités internationales et ce, depuis 2008 : "On peut considérer les MOOC comme une nouvelle forme d'éducation à distance dont la popularité dans les universités d'Amérique et d'ailleurs peut être qualifiée de phénoménale ces dernières années » (Karsenti, 2013, p. 7). Ainsi, les universités marocaines surfent sur cette vague et misent sur cette « innovation » pédagogique pour redorer leur image, gagner en visibilité à l'international et diversifier leurs offres de formation. Un autre avantage, propre aux MOOCs et qui répond à une problématique nationale, est celui du nombre impressionnant d'étudiant-es qui peuvent participer aux MOOCs : «Sur le plan pédagogique, les universités peuvent surmonter le défi de la massification grâce aux technologies appropriées, concevoir des MOOC, assurer le 
tutorat des étudiants, concevoir des enseignements à distance (initiale et continue) » (Conseil Supérieur de l'Éducation, 2018, p. 89). Ainsi, tout en considérant que le numérique est en phase embryonnaire dans nos établissements, des partenariats ont été menés afin de promouvoir des projets portant sur la conception de MOOCs et leur dérivé, les Small Private Online Course (SPOC). Nous citons les plus importants, comme le projet MarMOOC lancé en 2017, ayant pour objet le partenariat avec les pays du Nord dans le cadre d'Erasmus+. Ce projet concerne 14 partenaires marocains et européens réunis au sein d'un consortium. Un autre projet important est le projet MUN. II s'agit d'un accord visant la création de la plateforme "Maroc Université Numérique », à l'image de "France Université Numérique », et qui a été signé, en 2016, entre le Ministère de l'Enseignement Supérieur Marocain, France Université Numérique et l'Ambassade de France au Maroc. En effet, l'intérêt des deux projets est, d'une part, de former les enseignant-es aux pratiques numériques et d'autre part, de partager et diffuser les différentes expériences des universités marocaines conduites dans le cadre des MOOCs et SPOCs et ce, sur une seule plateforme nationale. Ceci permettra notamment de mutualiser les ressources et faire profiter un grand nombre d'apprenant-es/étudiant-es.

\section{PROBLÉMATIQUE ET OBJECTIF DE LA RECHERCHE}

Nous avons relevé que les efforts fournis pour promouvoir le numérique restent axés uniquement sur les volets « conception » et « mise en place » c'est-à-dire dans une approche techno-centrée et non apprenant-centrée. En effet, et jusqu'à présent il y a eu très peu de recherches académiques et d'études scientifiques nationales portant sur l'apport du numérique pour les étudiant-es marocains. Ainsi, se focaliser sur l'étudiant en adaptant une approche apprenant-centrée devient un besoin en recherche important, car ce qui importe, in fine, est l'apport réel du numérique pour l'éducation. II s'agit ainsi de comprendre comment le numérique se voit appréhendé par les apprenant-es et comment elles et ils utilisent et s'approprient cette technologie dans leur vie quotidienne d'apprenant-es. En effet, nous mettons l'accent sur une problématique touchant plus particulièrement les étudiant-es inscrits aux établissements à accès ouvert, à savoir le manque de motivation (Conseil Supérieur de I'Éducation, 2018). De par notre position d'enseignant-e à la Faculté des Sciences de Rabat (FSR), nous avons cherché à apporter des solutions à la non-motivation des étudiant-es que nous suivons dans le cadre du module accrédité « Langue et Terminologie ». Une des solutions qui semble porter un apport positif sur le construit de la motivation en éducation est l'intégration des pratiques numériques dans l'enseignement-apprentissage.

Ainsi, l'objectif de cette recherche est de comprendre l'impact réel d'un dispositif de formation de type SPOC sur la motivation autodéterminée des étudiant-es universitaires marocain-es, afin de sortir avec des éléments de réponse, inspirés et relevés d'une expérience à la FSR. Pour y parvenir, nous avons conçu un SPOC. Sur le même modèle que les MOOCs, les SPOCs s'en distinguent au niveau du public visé, volontairement limité à un effectif réduit de participant-es partageant les mêmes attentes, ce qui facilite l'accompagnement et le suivi des apprenant-es. Les résultats obtenus dans le cadre de cette recherche nous permettront de savoir si les 
étudiant-es universitaires marocain-es sont plus motivé-es à apprendre en contexte d'apprentissage en ligne. De tels résultats peuvent aider à positionner et orienter la stratégie universitaire marocaine en matière de numérique en général et de SPOC en particulier.

\section{LA QUESTION DE LA MOTIVATION DANS LA LITTÉRATURE}

\subsection{Qu'est-ce que la motivation?}

Lors de nos recherches sur la motivation, nous avons constaté qu'il existe autant de définitions que d'auteurs. Cependant, les termes « action », « comportement » et « objectif » attirent notre attention car ils sont souvent abordés par les différent-es chercheurs et chercheuses.

Pour Vallerand et Thill (1993), « le concept de motivation représente le construit hypothétique utilisé afin de décrire les forces internes et/ou externes produisant le déclenchement, la direction, l'intensité et la persistance du comportement » (p. 18).

Selon Fenouillet (2012) « il n'existe pas une seule forme de motivation. La motivation est avant tout un terme générique, généralement utilisé à défaut d'une spécification plus précise sur la nature exacte de la force qui produit un comportement ou une action (p. 27).

Pour l'approche de la psychologie cognitive, la motivation est « une hypothétique force intraindividuelle protéiforme, qui peut avoir des déterminants internes et/ou externes multiples, et qui permet d'expliquer la direction, le déclenchement, la persistance et l'intensité du comportement ou de l'action » (Fenouillet, 2012, p. 9).

En contexte éducatif, la motivation semble être le premier pas vers la réussite et l'accomplissement scolaire. Pour Viau (1994), la motivation est « un phénomène qui tire sa source dans des perceptions que l'apprenant a de lui-même et de son environnement, et qui a pour conséquence qu'il choisit de s'engager à accomplir l'activité pédagogique qu'on lui propose et de persévérer dans son accomplissement, et ce, dans le but d'apprendre » (p. 31).

Ainsi, la motivation est une force interne propre à chaque individu-e, qui le pousse à dépasser ses limites et à chercher au fond de lui des raisons (des motifs) de changer de comportement pour passer à l'action, afin d'aboutir à un résultat. En éducation, plusieurs auteurs (Karsenti, 1997 ; Viau, 1994 ; Viau, et al., 2004) s'accordent et affirment que la motivation est l'un des facteurs clés de la réussite scolaire. Elle contribue grandement à l'apprentissage des apprenant-es et dans l'accomplissement des objectifs. Par ailleurs, de nombreuses recherches ont montré que le manque de motivation est l'une des causes principales de l'absentéisme, de l'obtention de mauvaises notes et, éventuellement, de l'abandon scolaire/universitaire. Ainsi, il nous parait important de comprendre la motivation des étudiant-es inscrits à la FSR et plus particulièrement de celles et ceux inscrits en filière «Sciences de la Matière Physique Chimie » (SMPC). 


\subsection{La motivation autodéterminée}

Le cadre théorique mobilisé dans le cadre de cette recherche est une adaptation (Heutte, et al., 2016) des travaux de Deci \& Ryan (2000) portant sur la motivation à travers la théorie de l'autodétermination (TAD). Cette théorie est tout particulièrement intéressante pour la question de la motivation des apprenant-es qui participent à des MOOCs, puisqu'elle illustre le fait que certains types de la motivation extrinsèque ont également des impacts positifs sur la motivation à apprendre des apprenant-es (Karsenti, 2015). Les auteurs conçoivent trois grandes formes de motivation, organisées selon un continuum : la motivation intrinsèque, la motivation extrinsèque et l'amotivation. Chaque forme de motivation est associée à un niveau de comportement autodéterminé et autonome. La motivation intrinsèque (MI) est une motivation autonome égocentrée (Heutte, et al., 2016). A l'opposé de la Ml, se trouve l'amotivation (AM), qui est caractérisée par l'absence de motivation et une perception d'un manque de compétence (Bandura, 2003 ; Deci, 1985). Les comportements observés dans le cadre de la motivation extrinsèque (ME) correspondent à quatre types de régulation : externe, introjectée, identifiée et intégrée, allant du plus bas au plus haut degré de l'autodétermination. Elles sont classées et définies comme suit (Figure 1).

Figure 1. Schématisation des six types de régulations correspondant à chaque type de motivation

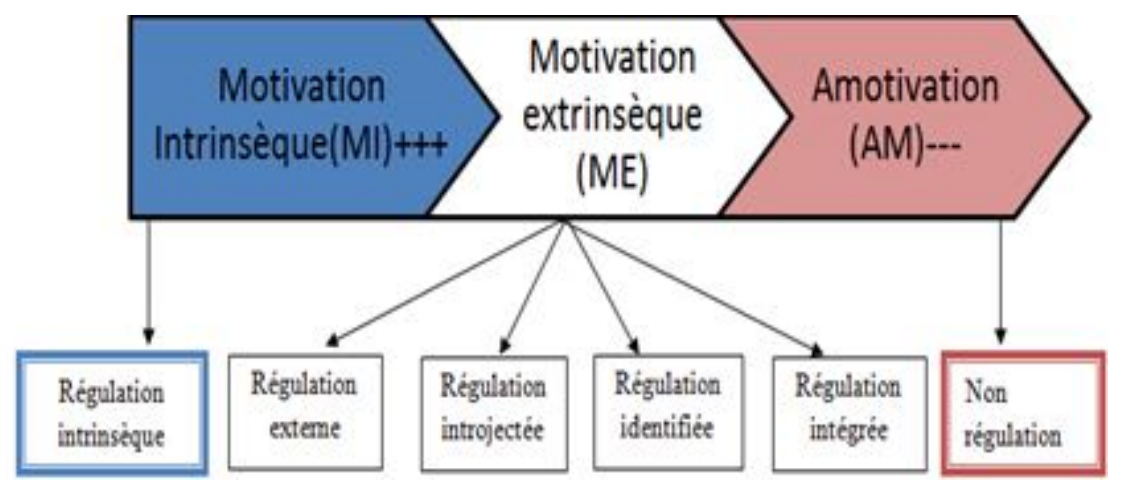

Source : Heutte, et al. (2016)

- Régulation externe : Les raisons du comportement trouvent leur justification dans des demandes ou contraintes externes (la recherche d'une récompense ou l'évitement d'une sanction). En contexte éducatif, ces demandes sont généralement liées aux demandes de l'enseignant-e/tuteur/trice.

- Régulation introjectée : Les raisons du comportement sont légèrement intériorisées, mais pas suffisamment pour être considérées comme faisant partie intégrante de soi (Deci \& Ryan, 2000). Les actions restent soutenues par des pressions internes telles que la honte ou la culpabilité.

- Régulation identifiée : A ce niveau, l'individu-e est intéressé par l'activité tout en étant conscient de la finalité escomptée par celle-ci : « Les raisons du comportement sont liées 
à une bonne compréhension des objectifs définis par un contexte social et par une acceptation consciente de la valeur des objectifs et de la nécessité des actions à entreprendre pour les atteindre " (Deci \& Ryan, 2000, cités par Heutte, et al., 2016, p. 101).

- Régulation intégrée: Cette régulation représente le degré le plus élevé d'autodétermination de la motivation extrinsèque. L'individu-e est engagé dans l'action car celle-ci correspond à ses attentes et aspirations :

La régulation est interne, tout en étant en concordance avec les valeurs du comportement définies par un contexte fortement marqué par le sentiment d'appartenance sociale (Ryan \& Deci, 2000) : il s'agit d'une motivation autonome allocentrée. Ces effets sur le bien-être et la santé mentale sont comparables à ceux qui sont observés au niveau de la motivation intrinsèque. (Heutte, et al., 2016, p. 101).

Les régulations externes et introjectées correspondent à une motivation contrôlée par des demandes externes (demande de l'enseignant-e) tandis que les régulations identifiées, intégrées et intrinsèques renvoient à une motivation autonome. Ainsi, le seuil d'autodétermination entre la régulation introjectée et la régulation identifiée permet de distinguer la motivation contrôlée de la motivation autonome (Heutte, et al., 2016). Dans cet angle d'analyse, la TAD nous permettra, dans le cadre de cette recherche, de vérifier si la motivation des étudiant-es suivant un SPOC dans le cadre du cursus universitaire est autonome ou contrôlée. En d'autres termes, nous cherchons à comprendre la motivation de ces étudiant-es inscrits dans un cours en ligne en mettant l'accent sur l'apport de ce dernier sur la motivation à apprendre des apprenant-es pour enfin savoir si l'intégration du numérique susciterait l'autonomie des apprenant-es pour atteindre un niveau élevé d'autodétermination.

En effet, en contexte d'enseignement-apprentissage, la motivation est à la fois une source, par l'influence des perceptions de l'apprenant et une conséquence de la réussite (Viau,1994). Afin de comprendre la motivation en contexte scolaire/universitaire, il faut s'arrêter à une composante importante de la motivation, à savoir l'engagement. Ainsi, nous étudions, dans un paradigme sociocognitif, le concept de l'engagement étant directement considéré comme un indicateur de motivation (Viau, et al., 2004).

\subsection{L'impact du numérique sur la motivation et l'engagement des apprenant-es}

La documentation scientifique a abordé l'apport du numérique comme facteur motivationnel chez les apprenant-es : "Les TIC semblent améliorer les connaissances, les aptitudes et les compétences transversales, en contribuant à la motivation, au plaisir d'apprendre et à l'estime de soi »(Loisier, 2011, p. 67). Aussi, l'intégration des TIC peut aider à créer un contexte d'apprentissage susceptible de favoriser l'engagement (Blumenfeld, et al., 1991).

Marchand (2001) a mené une étude sur l'usage du numérique (TIC) en éducation au Canada. Elle distingue des avantages et des contraintes concernant l'apprenant-e, l'enseignant-e et l'institution, associés à l'apprentissage en ligne dans le contexte universitaire. De son étude, il 
se dégage que les avantages du numérique (par ex., interactions plus faciles entre pairs et avec l'enseignant-e, disponibilité du contenu, flexibilité dans l'apprentissage, meilleure gestion du temps, immersion technologique) dans les pratiques universitaires sont nombreux et semblent être plus importants que les contraintes (par ex., difficulté d'adaptation, isolement, coût du matériel). En effet, lorsque l'étudiant est mis en perpétuel contact avec le numérique, comme pour un cours en ligne, il développe plusieurs compétences :

L'étudiant doit apprendre à prioriser ses études, à choisir ses stratégies d'apprentissage, à organiser son horaire, à choisir l'information pertinente parmi les ressources offertes, à analyser, à observer et à critiquer ses actions, à vérifier son rythme de progression, à nommer ses difficultés et surtout à maintenir sa motivation tout au long de l'apprentissage ... ces compétences qui seront par la suite transférables de l'université au lieu de travail. (Marchand, 2001, p. 416)

En définitive, l'utilité du numérique en pédagogie semble reconnue, tant par les enseignant-es que par les chercheurs et les chercheuses, comme une voie prometteuse pour améliorer la motivation et l'engagement des étudiant-es lorsque son intégration est conçue en prenant en considération le contexte et les attentes spécifiques des apprenant-es. En effet, l'intégration du numérique dans le contexte de l'enseignement-apprentissage à l'université permettrait aux étudiant-es de développer des capacités susceptibles d'accroitre leur motivation par le travail, en collaboration avec les pairs par des moyens variés et nouveaux, tout en développant des stratégies d'autonomie et d'adaptation dans l'apprentissage.

Ainsi, par ce travail, nous cherchons à exploiter d'autres moyens d'enseignementsapprentissages en l'occurrence le numérique pour motiver les étudiant-es marocain-es inscrites à l'université. Chemin faisant, nous chercherons plus spécifiquement à comprendre l'impact réel des SPOCs sur la motivation et l'engagement des étudiant-es universitaires marocain-es afin de sortir avec des éléments de réponse, inspirés et relevés d'une expérience à la FSR.

\section{CONTEXTE DE L’ÉTUDE : DÉVELOPPEMENT DU SPOC}

Les raisons derrière la création de ce SPOC sont nombreuses. Premièrement, il s'agit d'étudier la motivation autodéterminée des étudiant-es engagés dans un dispositif de type SPOC. Deuxièmement, il s'agit d'observer et d'analyser comment les étudiant-es universitaires marocain-es réagissent dans le cadre de l'apprentissage à distance. II est à noter que les étudiant-es passent d'un système scolaire arabisant à un système universitaire francisant. Cette transition, soudaine et non encadrée, constitue un grand problème, voire même un handicap pour la majorité des étudiant-es dans leur cursus universitaire.

Ainsi, nous avons conçu, avec l'appui et le soutien du doyen de la Faculté des Sciences, en partenariat avec le E-learning Center de l'Université Mohamed V de Rabat, un SPOC intitulé « Initiation à la Terminologie Scientifique en langue française » (ITS) et diffusé sur la plateforme de l'Université mooc.um5.ac.ma. En suivant les démarches d'élaboration d'un cours de 
Français sur Objectif Spécifique (FOS), le SPOC était conçu et destiné aux étudiant-es inscrits en filière Science de la Matière Physique Chimie (SMPC), en prenant en considération le contexte spécifique de ces étudiant-es voulant poursuivre leurs études en langue française dans une discipline scientifique. L'inscription au SPOC ITS était obligatoire, car ce dernier comptait dans la notation du module «Langue et Terminologie ", à raison de $30 \%$ de la note finale. II a été lancé le 4 septembre et a pris fin le $1^{\text {er }}$ octobre 2017. Chaque semaine était dédiée à une thématique scientifique relative aux modules enseignés lors du premier semestre.

Nous nous sommes basés sur nos observations, étant professeure de langue et terminologie, tout en prenant en considération tous les besoins et attentes exprimés par les étudiant-es de la filière SMPC dans la réalisation du scénario (Figure 2).

\title{
Figure 2. Scénarisation du SPOC Initiation à la Terminologie Scientifique (ITS)
}

\author{
Semaine 0 : Présentation du SPOC \\ Objectif de la semaine : Familiariser les étudiants avec la plateforme OpenEdx \\ Video1 : Présentation du SPOC \\ Forum : Discussion et partage de contact \\ Semaine 1: La physique, c'est fantastique
}

Objectif de la semaine : Introduction à la thermodynamique. La vidéo 2 mettra l'accent sur l'explication et la traduction de la terminologie abordée par le professeur (vidéo 1).

Forum : Discussion entre pairs sur la thématique

Document à télécharger : le lexique scientifique Français-Arabe.

Vidéo1 : Introduction à la thermodynamique 1

Vidéo 2 : Terminologie de l'introduction à la thermodynamique 1

Évaluation : Quiz

Semaine 2 : A la découverte de la chimie

Objectif de la semaine : La découverte de la chimie et plus précisément la thermochimie et l'atomistique. Une attention particulière sera dédiée à l'explication et à la traduction des mots techniques.

Forum : Discussion entre pairs sur la thématique

Documents à télécharger : Liste des préfixes scientifiques / liste des suffixes scientifiques

Vidéo 1 : Introduction à la thermochimie

Vidéo 2 : Terminologie de l'introduction à la thermochimie

Évaluation : Quiz 1

Vidéo 3 : Introduction à l'atomistique

Vidéo 4 : Terminologie de l'introduction à l'atomistique

Évaluation : Quiz 2

Semaine 3 : Bienvenue au monde d'Al Khwarizmi

Objectif de la semaine : Initier les étudiants à la terminologie des modules «Algèbre et Analyse ».

Forum : Discussion entre pairs sur la thématique

Vidéo 1 : Terminologie de l'introduction à l'Algèbre et l'Analyse

Évaluation : 1) Poster sur la plateforme un exercice d'analyse en langue française

2) Évaluer les pairs (à partir d'une grille d'évaluation)

Semaine 4 : Comment réviser, mémoriser et apprendre efficacement ?

Objectif de la semaine : Initiation aux techniques de méthodologie universitaire.

Documents/ ressources à télécharger : a) Deux textes ; b) Exemple de sketchnote b) une vidéo (contenu portant sur les thématiques abordées au cours des semaines écoulées).

Forum : Discussion entre pairs

Vidéo 1 : Explication des trois techniques "carte mentale, prise de notes, sketchnote "

Évaluation finale : Poster sur la plateforme le devoir final (pratiquer les trois techniques expliquées dans la vidéo)

Comme acteurs/trices, décideurs et décideuses du SPOC ITS, nous avons : 
- Six professeur-es de discipline (Mathématiques, Sciences et Physique), qui ont fourni les thématiques et le contenu scientifique ;

- Une professeure de "Langue et terminologie » qui a assuré la coordination et la conception du SPOC ;

- L'équipe du E-learning Center de Rabat qui a apporté de l'aide en matière de logistique, d'enregistrement des vidéos et d'intégration sur la plateforme OpenEdx.

La session 1 était une expérience inédite en matière de dispositif d'apprentissage numérique pour l'ensemble des acteurs/trices (responsables à la FSR, enseignant-e-es/tuteurs/trices, étudiant-es). Nous avons pu mener à bon port le SPOC avec 792 inscrit-es.

\section{MÉTHODOLOGIE}

Nous avons opté pour une démarche de recherche mixte concrétisée par l'utilisation d'une échelle de motivation afin de recueillir des données d'opinion par rapport l'expérience du SPOC ainsi que l'analyse des traces des apprenant-es sur la plateforme. Notons que ces données sont croisées pour apporter plus de précisions au niveau des résultats.

Notre échantillon est constitué d'étudiant-es inscrit-es en première année à la FSR pour l'année universitaire 2017/2018 en filière SMPC, soit un effectif de 792 étudiant-es. Un questionnaire, basé sur l'Échelle de Motivation en Formation d'Adultes (EMFA) développée par Fenouillet, Heutte et Vallerand (2015) et sur la théorie de l'autodétermination de Deci et Ryan, a été administré en ligne sur Google Forms aux étudiant-es en fin du SPOC. Le choix d'opter pour l'EMFA est justifié par plusieurs raisons. D'abord, il s'agit d'une mesure de la motivation en formation des adultes. Ensuite, elle propose de mesurer la motivation des adultes dans le contexte d'un dispositif de formation (MOOC/SPOC). Enfin, elle ajoute une mesure de la régulation intégrée qui est la forme la plus autodéterminée de la motivation extrinsèque, et qui prend particulièrement son sens dans le cadre de la formation des adultes.

Cette échelle mesure 6 différents construits, la motivation intrinsèque à la connaissance, la régulation externe, introjectée, identifiée, intégrée et enfin l'amotivation. Elle contient 24 énoncés (4 énoncés par sous-échelle) et est mesurée sur une échelle de 1 à 7 points de Likert allant de 1 (Ne correspond pas du tout) à 7 (Correspond très fortement). Le tableau 1 cidessous fournit un exemple d'un item pour chaque type de motivation. 
Tableau 1. Exemple d'item pour chaque type de motivation

\begin{tabular}{|l|l|}
\hline \multicolumn{1}{|c|}{ Type de motivation } & \multicolumn{1}{c|}{ Exemple d'items } \\
\hline Motivation intrinsèque à la connaissance & $\begin{array}{l}\text { Parce que j'éprouve du plaisir et de la } \\
\text { satisfaction à apprendre de nouvelles } \\
\text { choses. }\end{array}$ \\
\hline Motivation extrinsèque - Intégrée & $\begin{array}{l}\text { Parce que ce type de formation fait partie } \\
\text { intégrante de moi. }\end{array}$ \\
\hline Motivation extrinsèque - identifiée & $\begin{array}{l}\text { Parce que selon moi elle va m'aider dans } \\
\text { la poursuite de ma carrière (ou pour ma } \\
\text { future carrière) }\end{array}$ \\
\hline Motivation extrinsèque - introjectée & $\begin{array}{l}\text { Pour me prouver à moi-même que je suis } \\
\text { capable de suivre cette formation. }\end{array}$ \\
\hline $\begin{array}{l}\text { Motivation extrinsèque - régulation } \\
\text { externe }\end{array}$ & $\begin{array}{l}\text { Parce qu'elle va me permettre de gagner } \\
\text { davantage }\end{array}$ \\
\hline Amotivation & $\begin{array}{l}\text { Honnêtement, je ne le sais pas ; j'ai } \\
\text { vraiment l'impression de perdre mon } \\
\text { temps en formation. }\end{array}$ \\
\hline
\end{tabular}

En termes des limites méthodologiques de notre recherche, l'outil quantitatif mobilisé soit l'échelle (EMFA) aborde une notion si personnelle, soit la motivation, et repose sur des expériences menées dans des pays occidentaux. Étant donné qu'il n'y a pas eu une contextualisation de l'échelle, la question de sa validité d'un point de vue culturel au contexte de recherche se pose.

Par ailleurs, il aurait été intéressant d'administrer le questionnaire auprès des mêmes étudiantes, à différents temps de leur formation pour observer leurs «trajectoires » de motivation et ainsi apprendre davantage sur leur motivation en action. Mais, il faut rappeler qu'il s'agit d'une échelle de 24 items, rédigée en langue française, destinée à des étudiant-es arabisé-es n'ayant jamais répondu à un questionnaire auparavant. D'ailleurs, nous avons été amenée à traduire oralement en langue arabe les items de notre questionnaire pour en simplifier la compréhension et pour assurer une crédibilité dans les réponses.

\section{RÉSULTATS}

\subsection{Données de l'enquête}

Sept cent quatre-vingt-douze étudiant-es se sont inscrits au SPOC soit $87 \%$ des étudiant-es inscrit-es au premier semestre de l'année universitaire 2017/2018, 604 étudiant-es ont été actifs et actives sur la plateforme (elles et ils consultaient les documents, vidéos, quiz ainsi que la tâche finale). Quatre cent trois sur les 792 étudiant-es, soit 51\% ont obtenu une note supérieure à 50\%. 
Au total, 244 étudiant-es (144 femmes et 100 hommes), soit un peu moins d'un tiers, ont renseigné le questionnaire qui leur a été administré à la fin du SPOC. Pour pouvoir mener les analyses statistiques nécessaires à notre étude, nous avons procédé dans un premier temps à calculer l'indice d'autodétermination (IAD) 1 pour chacun des répondant-es à l'EMFA. Plus le score de cet indice est élevé, plus le degré d'autodétermination de la motivation est fort, " ce qui représente la perception de la proactivité, du choix, de la liberté d'agir, de l'autonomie par le sujet lui-même » (Carré, 2005, p. 140). En d'autres termes, l'indice de l'IAD (Tableau 2) indique si la motivation des répondant-es est autonome ou contrôlée.

\section{Tableau 2. Score de l'indice d'autodétermination}

\begin{tabular}{|l|l|}
\hline Indice (IAD ) N=244 & 3,57 \\
\hline Score supérieur & 36 \\
\hline Score inférieur & -36 \\
\hline
\end{tabular}

Le résultat de cet indice, légèrement supérieur à la moyenne et qui doit être supérieur à 0 , indique que le taux de IAD est faible ce qui laisse croire que la motivation des étudiant-es inscrits au SPOC ITS n'est pas autodéterminée. Autrement dit, elle n'est pas autonome (Deci \& Ryan, 2000).

a) Quel type de régulation ?

Pour confirmer ce que I'IAD semble montrer, nous avons cherché à vérifier si la motivation autodéterminée des étudiant-es inscrit-es au SPOC ITS a un lien significatif avec la régulation intégrée de la motivation (MMEIN), qui représente la motivation optimale en contexte institutionnel (Heutte, et al., 2016). Pour ce faire, nous avons procédé au classement des six types de motivation (Figure 3).

\footnotetext{
${ }^{1}$ Le calcul est le suivant : IAD $=(3 \times$ Motivation intrinsèque à la connaissance $)+(2 \times$ Régulation Intégrée de la Motivation extrinsèque) + (Régulation Identifiée de la Motivation extrinsèque) - (Régulation Introjectée de la Motivation extrinsèque) - (2 $x$ Régulation Externe de la Motivation extrinsèque) - (3 x Amotivation) (Fenouillet, et al., 2015)
} 
Figure 3. Boîte à moustache des dimensions de l'EMFA

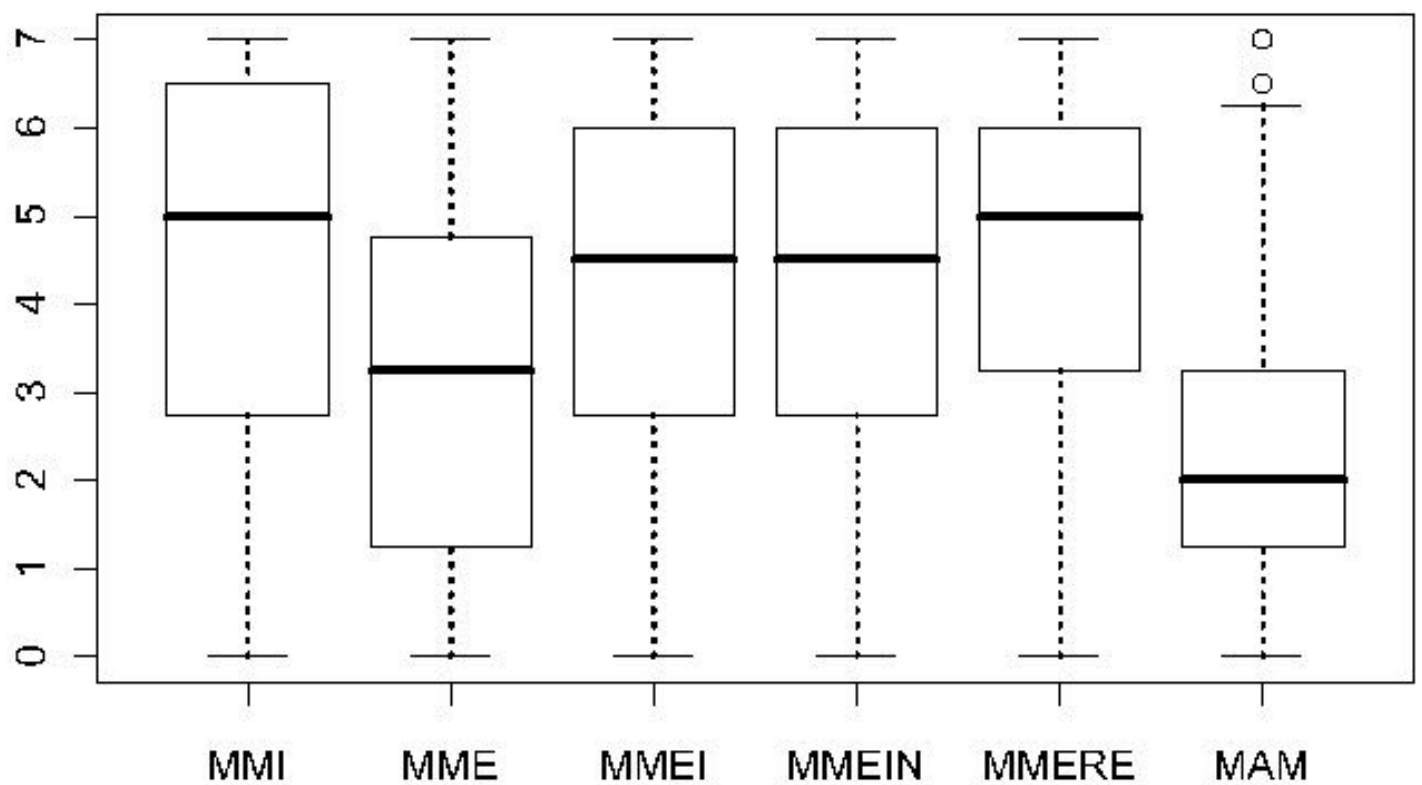

- Pour la variable MMl, la médiane des scores obtenus est de 5. Le quart de l'échantillon (25\% des étudiant-es) a une motivation faible avec des scores se situant entre 0 et 2.75. Tandis que les scores obtenus par les étudiant-es ayant une motivation forte, se situent entre 5 et 7 . La distribution est plus allongée vers les valeurs à motivation faible.

- Pour la variable MME, la médiane des scores obtenus est de 3.250. Le quart des étudiant-es a une motivation faible avec des scores se situant entre 0 et 1.250. Les scores obtenus par les étudiant-es ayant une motivation moyenne se situent entre 1.250 et 3.250 tandis que les scores obtenus par les étudiant-es ayant une motivation forte se situent entre 3.250 et 7. La distribution est plus allongée vers les valeurs à motivation forte.

- Pour les variables MMEl et MMEIN, la médiane des scores obtenus est de 4.500. Le quart des étudiant-es a une motivation faible avec des scores se situant entre 0 et 2.750. Les scores obtenus par les étudiant-es ayant une motivation moyenne se situent entre 2.750 et 4.500 tandis que les scores obtenus par les étudiant-es ayant une motivation forte se situent entre 4.500 et 7 . La distribution est plus allongée vers les valeurs à motivation moyenne.

- Pour la variable MMERE, la médiane des scores obtenus est de 3.250. Le quart des étudiant-es a une motivation faible avec des scores se situant entre 0 et 3.250. Les scores obtenus par les étudiant-es ayant une motivation moyenne se situent entre 3.250 et 5 tandis que les scores obtenus par les étudiant-es ayant une motivation forte se situent entre 5 et 7 . La distribution est plus allongée vers les valeurs à motivation faible.

- Pour la variable MAM, la médiane des scores obtenus est de 2.000. Le quart des étudiant-es a une motivation faible avec des scores se situant entre 0 et 1.250. Les scores obtenus par les étudiant-es ayant une motivation moyenne se situent entre 1.250 et 2 tandis que les scores obtenus par les étudiant-es ayant une motivation forte se situent entre 2 et 6.5 . 
Les indicateurs de cette boîte à moustache montrent que le type de motivation qui se distingue est la motivation extrinsèque à régulation externe (MMERE). Cette régulation contrôlée est caractérisée par un comportement lié à des demandes externes ou à des contraintes sociales, comme le fait de souhaiter s'inscrire en formation parce que la participation à cette formation peut permettre d'éviter une sanction (une mauvaise note) ou d'obtenir une récompense (une promotion, une meilleure note).

Dans notre contexte, nous pouvons expliquer ce résultat par le fait que les étudiant-es ont suivi le SPOC à la suite de raisons externes. II y a d'une part, la demande de l'enseignant-e à suivre le SPOC car cela rentrait dans le programme du module, et d'autre part parce que le cours était récompensé par une note. Ainsi le fait de suivre le cours permettait aux étudiant-es d'éviter la sanction, soit la non-validation du module.

b) Quel lien entre l'outil de travail et la motivation autodéterminée ?

Nous avons constaté que la quasi-totalité des étudiant-es accédaient au cours via leur téléphone portable, une décision qui ne se présentait pas par choix mais par obligation, plus précisément par manque de moyens. Notons au passage que la plateforme du cours OpenEdx n'était pas adaptée au téléphone, c'est-à-dire, qu'il n'y avait pas la possibilité de télécharger une application conçue pour téléphone. Ainsi, nous avons cherché à comprendre le lien entre l'outil de travail et la motivation des étudiant-es.

Une analyse des graphes des individu-es (Figure 4) nous indique que le quadrant sud-est représente les régions des étudiant-es les plus motivés et les plus dynamiques. Cette catégorie est représentée majoritairement par des femmes avec l'utilisation des ordinateurs et avec très peu de difficultés rencontrées. 
Figure 4. Graphes des individu-es

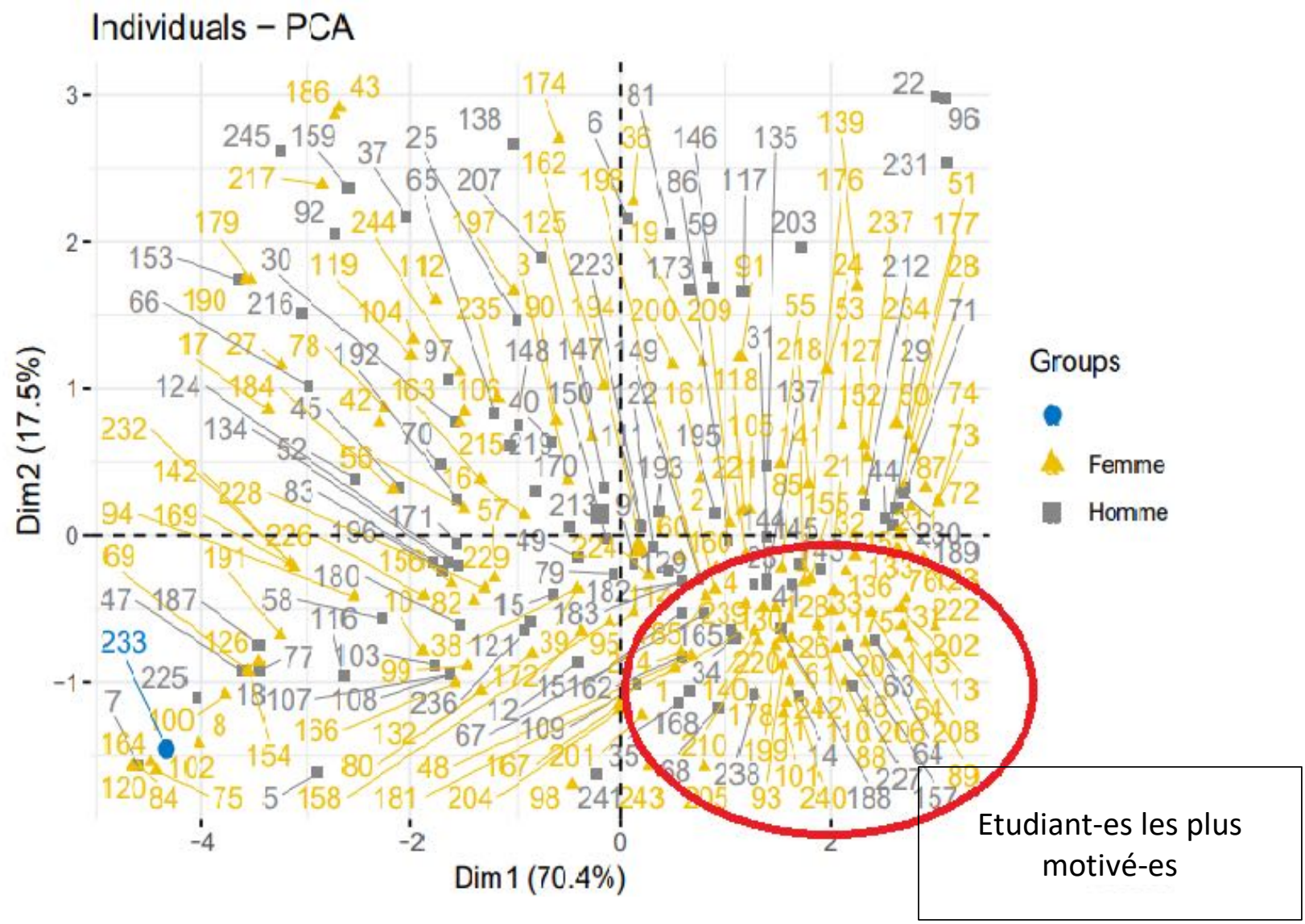

Individuals - PCA

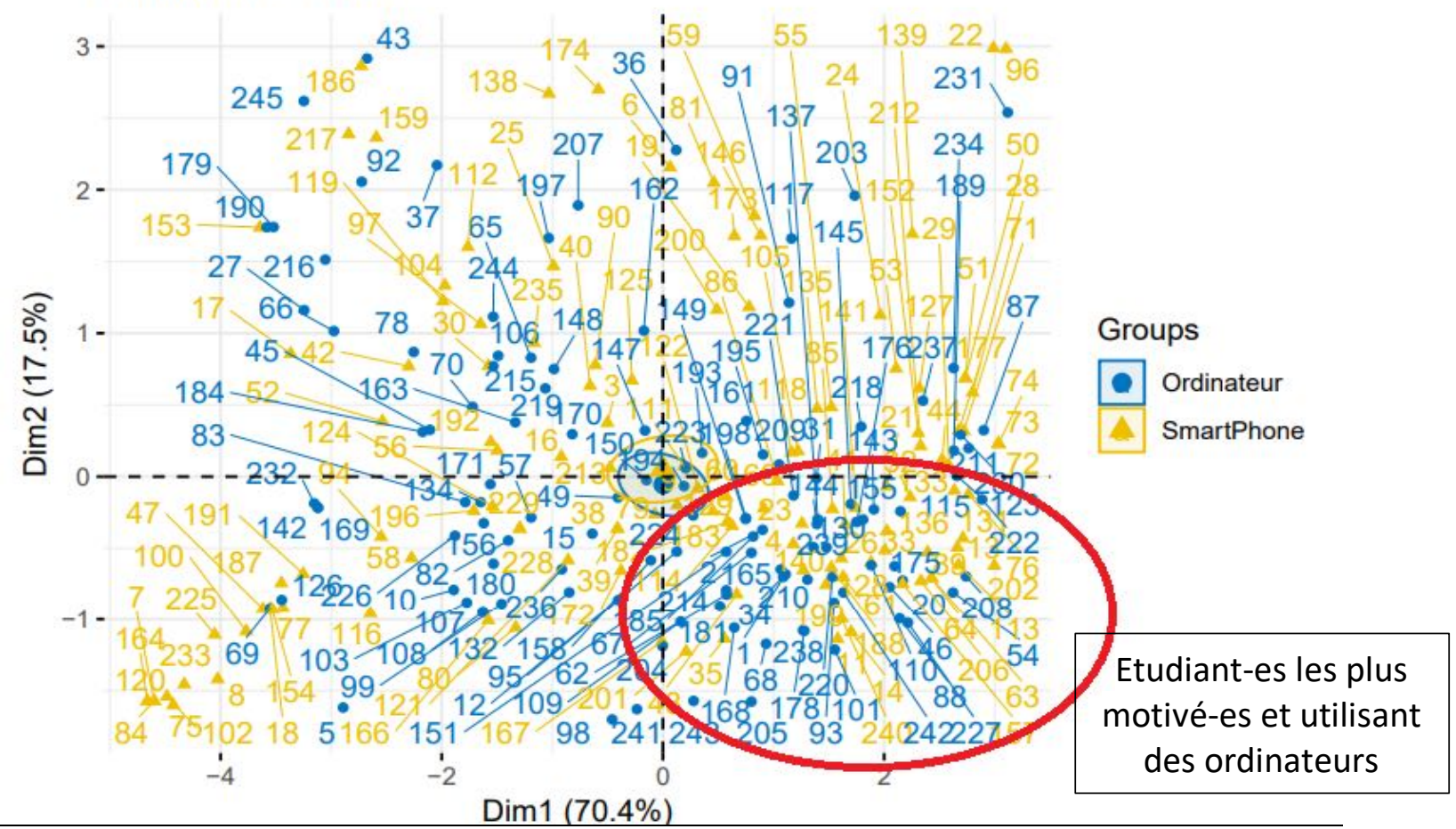


Ainsi, les étudiantes/femmes se sont déclarées plus motivées que les étudiants homme. Elles utilisent un ordinateur pour suivre le SPOC ITS, ce qui rend le suivi du cours et la réalisation des activités plus facile et plus pratique. Par conséquence, elles ne rencontrent pas de problèmes relatifs à la plateforme. En revanche, les étudiants/hommes accèdent au cours via leur téléphone. Étant donné que la version de la plateforme OpenEdx qui héberge le SPOC ITS n'est pas adaptée aux téléphones, ces étudiants rencontrent des problèmes d'ordre technique et ergonomique (par ex. visionnage des capsules vidéo, évaluation par les pair-es, réalisation de l'activité finale sur Word et PDF). Ces problèmes ont constitué des obstacles influençant négativement leur motivation.

II se dégage clairement des résultats quantitatifs que la motivation des étudiant-es à s'inscrire au SPOC ITS était contrôlée et non pas intrinsèque. Cependant, ces mêmes étudiant-es ont fait preuve d'un grand engagement et de persévérance dans le suivi du cours (appuyé par les traces laissées sur la plateforme). Chose qui laisse penser que le scénario pédagogique tel qu'il a été élaboré a suscité leur intérêt.

\subsection{Analyses des traces}

Nous nous sommes intéressés à analyser le comportement des étudiant-es en nous focalisant sur l'engagement comportemental - étant le type d'engagement le plus observable. Pour ce faire, nous nous sommes basés sur les travaux de Fredericks, et al. (2004) expliqué plus haut. Dans notre contexte, nous avons fait le choix de mobiliser deux indicateurs à savoir «la conduite » et "l'implication active » complétés par l'analyse des scores obtenus en fin de formation.

La conduite de la majorité des étudiant-es au sein de la plateforme répondait aux attentes fixées dans le scénario pédagogique du cours qui renvoient, entre autres, au respect des règlements relatifs aux dates de réalisation/dépôt des devoirs, consultations des documents et des ressources, visionnage des vidéos et participation au forum. En moyenne, les étudiantes ont consacré trois heures par semaine au SPOC ce qui correspond à la charge de travail estimée dans le cahier des charges du cours. Par ailleurs, les étudiant-es se sont impliqués activement dans le cours en persistant, avec les moyens limités qu'ils avaient, à réaliser toutes les tâches y compris celles qui exigeaient des efforts par exemple l'évaluation par les pairs ou l'activité finale. Pour passer au crible la conduite et l'implication des étudiant-es, nous mettons l'accent sur deux indicateurs :1) la participation au forum 2) les productions des étudiant-es (les rendus) à la suite de Fredricks, et al. (2004), Linnenbrink et Pintrich (2003).

a) Le forum

Le forum était le terrain de travail collaboratif à distance entre les étudiant-es, et ce, au travers des échanges portant sur les thématiques traitées chaque semaine et les activités qui s'y apparentent. En favorisant la communication entre les pairs et entre les enseignant-es, les étudiant-es ont pu dépasser leur timidité face à l'enseignant-e pour s'exprimer librement 
derrière leur identité virtuelle. Les échanges et le partage dans le forum étaient assez riches et beaucoup plus nombreux qu'en présentiel.

b) Les rendus des étudiant-es

Les trois techniques proposées dans la semaine cinq du SPOC ITS soit la carte mentale, la prise de note et le sketchnote avaient pour but de doter les étudiant-es de méthodes d'apprentissage, de synthèse et de mémorisation. Ainsi, après avoir visionné une capsule expliquant les règles et les démarches de réalisation de chaque technique, les étudiant-es devaient, individuellement, dans le cadre de l'évaluation finale, pratiquer les trois techniques. En termes de résultat, les productions des étudiant-es étaient au-delà de nos attentes d'enseignante - elles et ils ont laissé libre cours à leur imagination et créativité. Chaque étudiant-e a pu cerner les techniques à son rythme et à sa manière pour sortir avec des rendus de qualité. Le même devoir proposé en classe n'aurait pas abouti à de tels résultats avec autant de finesse et de richesse.

c) Les scores obtenus en fin de SPOC

En fin de formation, sur les 792 d'étudiant-es inscrit-es au SPOC, 403 ont réussi le cours soit un pourcentage de $51 \%$ dont 242 (soit 60\%) qui ont obtenu des scores supérieurs à 70/100 (ce score est rarement atteint en présentiel).

Tableau 3. Notes obtenues pour chaque semaine

\begin{tabular}{|c|c|c|}
\hline Semaine & $\begin{array}{c}\text { Ressources } \\
\text { d'évaluation }\end{array}$ & $\begin{array}{c}\text { Nombres } \\
\text { d'étudiant-es } \\
\text { ayant fait les } \\
\text { quiz/déposé un } \\
\text { devoir }\end{array}$ \\
\hline Semaine 1 & Quiz 1 & 652 \\
\hline Semaine 2 & Quiz 2 et 3 & 608 \\
\hline Semaine 3 & $\begin{array}{c}\text { Évaluation par les } \\
\text { pairs (exercice de } \\
\text { maths) }\end{array}$ & 341 \\
\hline Semaine 4 & Tâche finale & 434 \\
\hline
\end{tabular}

La lecture des scores (Tableau 3) relatifs à chaque semaine démontre que les étudiant-es ont maintenu une certaine présence et assiduité durant les cinq semaines du SPOC ITS ce qui a été traduit par la consultation de toutes les sections du cours (vidéos, documents, quiz, forum) et également par le score très élevé obtenu en fin de cours. 
En croisant les résultats de l'enquête par questionnaire et l'analyse des traces des étudiantes, il s'avère que les données se contredisent de manière forte. En d'autres termes, l'analyse des traces des étudiant-es sur la plateforme du SPOC ne reflètent pas les résultats de l'échelle EMFA. En effet, les données d'opinion exprimées par le biais du questionnaire restent plus faibles que les traces qui témoignent de l'action et montrent un engagement et une motivation élevés. Ainsi, les traces montrent que les étudiant-es ont été beaucoup plus motivés que ne le disent les résultats de l'enquête par questionnaire. Si la motivation extrinsèque à régulation externe (MMERE), étant une régulation contrôlée, est de fait la motivation qui se distingue dans le contexte de cette étude, l'engagement comportemental des étudiant-es durant les cinq semaines du cours va à l'encontre de ce résultat. D'ailleurs, ce dernier est en contradiction avec la théorie, puisque la régulation intégrée (MME) est la motivation optimale dans le contexte d'apprentissage scolaire via les dispositifs de formation numériques (Heutte, et al., 2016). Cela ouvre le débat par rapport à la validité de l'échelle dans notre contexte, d'autant plus que seul un tiers des étudiant-es a répondu au questionnaire.

\section{DISCUSSION ET CONCLUSION}

Des résultats de l'enquête, nous concluons que les étudiant-es n'ont pas réussi à se détacher de l'objectif externe de "note ", car les raisons de leur comportement n'étaient pas liées à une bonne compréhension et à une acceptation consciente des valeurs des actions induites pour l'atteinte des objectifs (Deci \& Ryan, 2000, cité par Heutte, et al., 2016). Autrement dit, suivre le SPOC pour son apport terminologique, linguistique et techno-pédagogique afin de cumuler un savoir leur permettant de mieux réussir leur insertion académique au sein de la FSR n'était pas leur priorité.

En effet, les explications que nous pouvons apporter à la motivation contrôlée de ces étudiantes renvoient à deux raisons. II y a d'un côté le système éducatif marocain qui, de l'école primaire jusqu'aux études supérieures, octroie une valeur importante à la note, qui est souvent considérée comme un indicateur de réussite ou d'échec. C'est d'ailleurs, cette « note » qui permet d'accéder ou pas aux grandes écoles - le seuil permettant de passer les concours des grandes écoles. Par conséquent, le rapport des étudiant-es à l'apprentissage devient conditionné par la récompense « note » au lieu de l'être par le savoir (Narcy-Combes \& NarcyCombes, 2019). D'un autre côté, le jeune âge des étudiant-es peut expliquer ce manque de motivation autodéterminée. Ces jeunes inscrits au premier semestre sont toujours en phase de transition post baccalauréat. Elles et ils n'ont pas été préparés à l'environnement universitaire, qui nécessite une autonomie et une proactivité dans l'apprentissage.

Notons aussi que dans le cadre de cette recherche, les étudiantes se sont démarquées par leur motivation. Elles accédaient au SPOC en utilisant un ordinateur ce qui leur a permis de suivre le cours dans de bonnes conditions, par conséquent, elles ont obtenu les meilleurs scores. En revanche, les étudiants suivaient le SPOC par téléphone, un choix qui peut être expliqué par le fait qu'ils soient plus mobiles privilégiant ainsi de suivre le cours par téléphone. 
Cependant, ce choix a impacté négativement leur motivation étant donné qu'ils ont souvent rencontré des problèmes d'ordre technique et ergonomique. II faudrait cependant investiguer plus finement les raisons pour lesquelles elles ont pu accéder à un ordinateur alors qu'ils ne l'ont pas fait ou pas pu le faire.

Les résultats relatifs à l'analyse des traces des étudiant-es révèlent qu'elles et ils ont manifesté une grande motivation durant les cinq semaines du cours, illustrée notamment par l'intérêt et l'implication active exprimés tout au long de la formation, ainsi que par le taux de réussite enregistré à la fin du SPOC. En considérant le modèle de Viau (1994) qui présente trois indicateurs de la compréhension de la motivation en contexte scolaire à savoir le choix d'entreprendre une activité d'apprentissage et non de l'éviter, la persévérance ou ténacité, et la performance ou résultats observables de l'apprentissage, nous pouvons avancer que la motivation autodéterminée des étudiant-es par rapport à l'inscription au SPOC était contrôlée. Cependant, leur comportement et engagement tout au long du cours, confirment l'impact positif d'un dispositif de formation de type SPOC sur leur motivation à apprendre à condition que le scénario pédagogique soit élaboré de telle manière à répondre à leurs attentes et besoins.

Par ailleurs, les débats autour d'un thème ou activité sont considérés par Linnenbrink et Pintrich (2003), comme un «instrumental help-seeking» qui est une forme d'appel à l'aide où l'apprenant-e demande de l'aide afin de comprendre et d'apprendre en allant au-delà de l'évaluation (quiz), ce qu'est considéré comme un signe positif d'engagement comportemental. En d'autres termes, le comportement des étudiant-es démontre les stratégies de profondeur adoptées par les étudiant-es qui ont dépassé les stratégies de surface, qui auraient été de se contenter du minimum demandé - faire uniquement le quiz pour obtenir la note. Le fait de chercher de l'explication et de la précision, traduit l'intérêt des étudiant-es pour enrichir leurs connaissances par rapport au contenu qui répond à leurs besoins spécifiques en matière de terminologie scientifique.

À la lumière de cette recherche menée dans le contexte universitaire marocain, cette expérience a permis la concrétisation de la possibilité de développer le numérique éducatif au sein des établissements d'enseignement supérieur. Ce grand saut dans l'enseignement à distance a entraîné également de nombreuses questions relatives à la massification, aux moyens et outils disponibles et enfin aux connaissances en informatique des enseignant-es et des étudiant-es. À la suite de la première séance, par exemple, et en se basant sur les retours des apprenant-es et des tuteurs/trices, des ajustements et des modifications ont été nécessaires pour améliorer le cours (par ex. simplification des modalités d'inscription à la plateforme, formation des tuteurs/trices, intégration de vidéos explicatives relatives à chaque activité, réorganisation des délais des évaluations). Nous pouvons avancer que le numérique éducatif peut être une solution à la massification dans la mesure où la programmation des cours à distance pourrait alléger le présentiel. Remplacer un cours magistral de thermodynamique, qui se déroule dans un amphithéâtre surpeuplé, où l'interaction avec 
l'enseignant-e est quasi inexistante, par un cours en ligne élaboré dans une approche de pédagogie active, permettrait d'accroitre la performance des étudiant-es. Cette meilleure performance serait possible grâce aux avantages offerts par le dispositif (par ex., flexibilité, liberté, temps de réflexion, visionnage illimité des vidéos), permettant ainsi à chaque étudiante d'envisager un apprentissage à son rythme.

Dans nos cours du module «Langue et Terminologie », à l'image des cours de langues étrangères, l'hétérogénéité des groupes constitue un sérieux problème. En programmant un cours en ligne dédié à celles et ceux qui ont un niveau avancé en langue permettrait, d'une part, de répondre à leurs attentes et perspectives - ce qui serait susceptible de les motiver et, d'autre part, de réduire l'effectif en présentiel en le limitant uniquement à celles et ceux du niveau inférieur.

Aussi, en intégrant le numérique dans nos facultés, nous offrons aux étudiant-es une immersion technologique et informatique qui les dotera de compétences susceptibles de leurs être utiles dans leur vie quotidienne d'apprenant-e pour une meilleure gestion et organisation de leurs apprentissages. Ces compétences seront par la suite transposées dans leur vie professionnelle, ce qui leur permettra d'être en harmonie avec les compétences du XXlème siècle.

Enfin, de nos résultats, nous avons relevé que l'expérience du dispositif de formation SPOC ITS a permis aux étudiant-es le développement de compétences transversales qu'ils avaient du mal à exprimer en classe. En effet, l'identité virtuelle a amené les étudiant-es au dépassement de soi. Les étudiant-es ont surmonté leur timidité et crainte en osant participer au forum, gagner en responsabilité, autonomie et créativité.

\section{RÉFÉRENCES}

Bandura, A. (2003). Auto-efficacité. Le sentiment d'efficacité personnelle. Bruxelles : De Boeck. Blumenfeld, P., Soloway, E., Marx, R. W., Krajcik, J. S., Guzdial, M., \& Pilinscar, A. (1991). Motivating project-based learning: sustaining the doing, supporting the learning. Educational Psychologist, 26(3-4), 369-398.

Bryan, C. L., \& Solmon, M. A. (2007). Self-determination in physical education: designing class environments to promote active lifestyles. Journal of teaching in physical education, 26, 260278.

Carré, P. (2004). Bandura : Une psychologie pour le XXlème siècle ? Savoirs, hors-série(5), 950. https://doi.org/10.3917/savo.hs01.0009

Carré, P. (2005). L'apprenance: Vers un nouveau rapport au savoir. Paris : Dunod.

Connell, J. P. (1990). Context, self, and action: a motivational analysis of self-system processes across the life span. In D. Cicchetti, \& M. Beeghly (Eds.), The self in transition: Infancy to childhood (pp. 61-97). Chicago, IL: University of Chicago Press. 
Conseil Supérieur de L’Éducation. (2018). L’Enseignement Supérieur Au Maroc : Efficacité, Efficience et Défis du Système. https://www.csefrs.ma/publications/lenseignementsuperieur-au-maroc/?lang=fr

Deci, E. L., \& Ryan, R. M. (1985). Intrinsic motivation and self-determination in human behaviour. New York: Plenum Press.

Deci, E. L., \& Ryan, R. M. (2000). Self-determination theory and the facilitation of intrinsic motivation, social development, and well-being. American Psychologist, 55, 68-78.

Fenouillet, F. (2012). Les théories de la motivation. Paris : Dunod.

Fenouillet, F., Heutte J., \& Vallerand R.-J. (2015, June 25-28). Validation of the adult education motivation scale. Fourth World Congress on Positive Psychology, Orlando, FL.

Heutte, J., Caron, P.-A., Fenouillet, F., \& Vallerand R. J. (2016). Étude des liens entre les caractéristiques instrumentales et les différents types de motivations des participants dans un MOOC. Revue Internationale Des Technologies En Pédagogie Universitaire, 13(23), 94110.

Fredricks, J. A., Blumenfeld, P. C., \& Paris, A. H. (2004). School Engagement: Potential of the Concept, State of the Evidence. Review of Educational Research, 74(1), 59-109.

Karsenti, T. (1997). Comment le recours aux TIC en pédagogie universitaire peut favoriser la motivation des étudiants : le cas d'un cours médiatisé sur le web. Cahiers de la Recherche en Éducation, 4(3), 455-484.

Karsenti, T. (2013). Les MOOC : Révolution ou simple effet de mode ? Revue Internationale Des Technologies En Pédagogie Universitaire, 10(2), 6-37.

Karsenti, T. (2015). MOOC : La pédagogie universitaire face aux MOOC. Revue Internationale Des Technologies En Pédagogie Universitaire, 12(1), 1-12.

Karsenti, T., \& Larose, F. (Eds.). (2001). Les TIC... au cœur des pédagogies universitaires : diversité des enjeux pédagogiques et administratifs. Québec, QC : PUQ.

Linnenbrink, E. A., \& Pintrich, P. R. (2003). The role of self-efficacy beliefs in student engagement and learning in the classroom. Reading and Writing Quarterly: Overcoming Learning Difficulties, 19(2), 119-137.

Loisier, J. (2011). Les nouveaux outils d'apprentissage encouragent-ils réellement la performance et la réussite des étudiants en FAD ? https://archives.refad.ca/recherche/TIC/TIC_et_reussite_des_etudiants.pdf

Marchand, L. (2001). L'apprentissage en ligne au Canada : frein ou innovation pédagogique? Revue Des Sciences De L'éducation, 27(2), 403-419.

Ministère de l'éducation nationale, de l'enseignement supérieur et de la recherche scientifique (2015). Rapport annuel 2015 des inspections générales. https://www.education.gouv.fr/Cid110456/Rapport-Annuel-2015-Des-InspectionsGenerales. Html

Narcy Combes J.-P., \& Narcy Combes M.-F. (2019). Cognition et personnalité dans I'apprentissage des langues : relier théories et pratiques. Paris : Didier.

Viau, R. (1994). La Motivation en contexte scolaire. St-Laurent, QC : Renouveau Pédagogique. 
Viau, R., Joly, J., \& Bédard, D. (2004). La motivation des étudiants en formation des maîtres à l'égard d'activités pédagogiques innovatrices. Revue Des Sciences de L'éducation, 301, 163-176.

Weinstein, C. E., \& Mayer, R. E. (1986). The teaching of learning strategies. In M. C. Wittrock (Ed.), Handbook of research on teaching (pp. 315-327). New York, NY: Macmillan.

Open Access Publications - Bibliothèque de I'Université de Genève Creative Commons Licence 4.0

$$
\text { (c) (1) }
$$

